\title{
Certificación turística sostenible y los impactos socioeconómicos percibidos por hoteles en Costa Rica
}

\author{
Sergio A. Molina-Murillo* \\ Universidad Nacional (Costa Rica)
}

\begin{abstract}
Resumen: En respuesta a los impactos negativos producto del rápido crecimiento del turismo en Costa Rica, el Gobierno creó el Certificado de Sostenibilidad Turística (CST). A través de este estudio se examina si los hoteles certificados perciben una mayor generación de beneficios socioeconómicos para sus comunidades locales y su importancia para optar por la certificación. Después de revisar la literatura, administrar seis entrevistas y realizar una prueba en 35 hoteles, se creó un cuestionario finalmente completado por representantes de 121 hoteles. El principal resultado muestra que el CST influye de manera favorable en la percepción de los impactos socioeconómicos positivos que los hoteles tienen en las comunidades donde operan, aunque estos parecieran ser efectos colaterales y no la razón principal para adoptar el CST.
\end{abstract}

Palabras Clave: Centroamérica; Comunidad local; Hotelería; Responsabilidad social empresarial; Turismo responsable.

Sustainable Tourism Certification and its Perceived Socio-economic Impacts in Costa Rican Hotels

Abstract: In response to the negative impacts of the accelerated growth of tourism in Costa Rica, the Government created the Certificate for Sustainable Tourism (CST). This study examines whether certified hotels perceive greater socioeconomic benefits as a result of the certification and therefore its importance for their local communities. After review of the existing literature, six interviews and a trial run carried out in 35 hotels, a questionnaire was finally completed by representatives of 121 hotels. The main result shows that the CST is seen positively as improving the socioeconomic impact that hotels have in the communities where they operate, although these seem to be collateral effects and not the mainstream reason for adopting CST.

Keywords: Central America; Environmental social responsibility; Hospitality; Local community; Responsible tourism.

\section{Introducción}

Promover el turismo responsable en destinos ambiental y socialmente frágiles debe ser una política prioritaria de los gobiernos y organizaciones del sector para resguardar el patrimonio sociocultural y ambiental. Costa Rica es considerado uno de los principales destinos de naturaleza con gran potencial de crecimiento (Flores, 2014; Honey, 2003); sin embargo, por el rápido crecimiento de la actividad turística (Figura 1) han surgido preocupaciones en el dominio púbico, en particular por la creciente presión sobre ecosistemas biodiversos (Hunt, Durham, Driscoll, \& Honey, 2015), así como impactos socioeconómicos negativos (Horton, 2009). A nivel general, tales impactos socioeconómicos de la actividad turística pueden manifestarse en la forma de fuga financiera, pérdida de la cultura local, y posibles desplazamientos de campesinos de sus tierras (p. ej., Smith 2015, Murray 2007). A nivel comunitario sus manifestaciones pueden ocurrir en la forma de inflación local, posible aumento de actividades como drogadicción o prostitución, o pérdida de la identidad local (p. ej., Andereck, Valentine, Knopf, \& Vogt, 2005).

Con el fin de abordar estas cuestiones ambientales y socioeconómicas, el Instituto Costarricense de Turismo (ICT) —una agencia gubernamental— creó en 1996 el Certificado de Sostenibilidad Turística

Universidad Nacional; E-mail: sergiomolina@una.ac.cr 
(CST), programa de certificación voluntario. A través de la participación en el programa - de hoteles u otros actores de la actividad turística- se busca una interacción balanceada entre el uso apropiado de los recursos naturales, el mejoramiento de la calidad de vida de las comunidades locales, y los rendimientos económicos de la actividad. De acuerdo a lo propuesto por Buckley (2012), se aboga por mejorar la forma en que se utilizan los recursos naturales y culturales, incentivar la participación activa de las comunidades locales, y gestionar de manera competitiva la actividad empresarial.

\section{Figura 1: Tendencias de la actividad turística en Costa Rica}

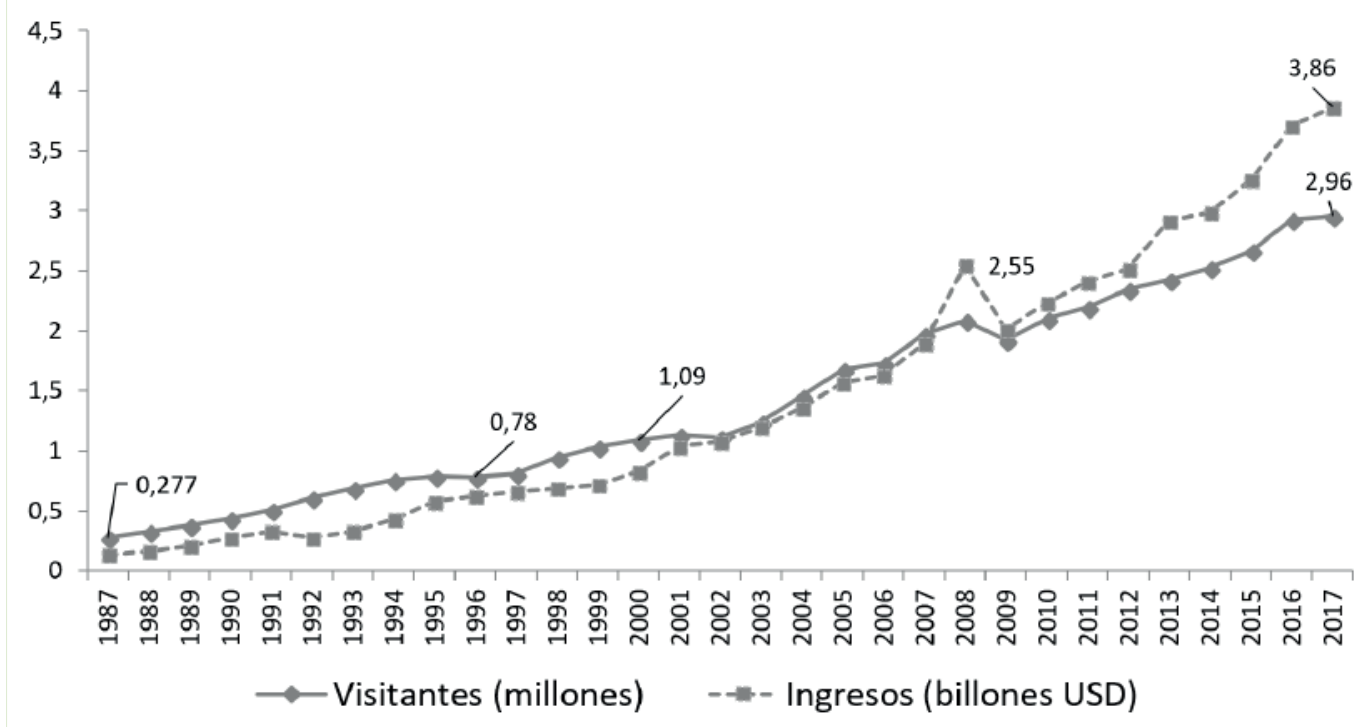

Fuente: Elaborado con base en información del ICT (2018a).

La mayoría de la investigación sobre este y otros programas de certificación en sustentabilidad se ha centrado en la dimensión ambiental en lugar de la socioeconómica (Vasconcelos-Vasquez, Balbastre-Benavent, \& Redondo-Cano, 2011) o se han realizado para casos específicos de un hotel (p. ej. Almeyda, Broadbent, Wyman, \& Durham, 2010; Vásquez \& Gutiérre, 2016). Entonces, el objetivo principal de este estudio es examinar la percepción sobre los impactos socioeconómicos positivos y negativos que los hoteles en Costa Rica — con o sin el Certificado para la Sostenibilidad Turística (CST) — pueden tener sobre sus comunidades de manera que se pueda propiciar una actividad turística más centrada en las poblaciones locales. ¿Perciben los hoteles certificados —en relación con los no certificados—una mayor generación de beneficios socioeconómicos para sus comunidades locales? ¿Es esta generación de beneficios a las comunidades realmente importante para optar por la certificación CST? Después del desarrollo de un marco contextual, se detalla la metodología, seguido por la presentación de los principales resultados obtenidos, y luego se finaliza con una discusión de los resultados y conclusiones.

\section{Turismo en Costa Rica}

Costa Rica es considerada una de las naciones con mayor biodiversidad del mundo. Ubicada entre América del Norte, América del Sur, y los océanos Pacífico y Atlántico, este país es caracterizado por un sistema montañoso, una marcada estacionalidad y temperaturas relativamente estables, que proveen numerosos y variados microclimas que sostienen a más de 500000 especies, cerca del $4 \%$ del total de las especies estimadas a nivel mundial en tan solo $0,03 \%$ de la superficie terrestre del planeta equivalente a $51100 \mathrm{~km} 2$ y $589000 \mathrm{~km} 2$ de mar territorial (INBio, 2016; Mok, 2005). Tal biodiversidad constituye un enorme potencial, ya sea con fines intelectuales, económicos o como instrumento para el desarrollo 
del país (INBio, 2016). A partir de la consolidación del sistema de áreas protegidas desde la década de 1970, junto con la condición de ser la democracia más estable de Centroamérica (la abolición de su ejército ocurrió en 1948), le ha permitido a Costa Rica posicionarse como uno de los ecodestinos más buscados del mundo (Lepree 2009; Lindberg y Aylward, 1999).

Durante los primeros años en los que el turismo internacional comenzó a expandirse, las agencias de ayuda internacional vieron en esta actividad un potencial para el crecimiento y el progreso para muchos países menos desarrollados. Para 1993 la actividad turística en Costa Rica había superado las otras exportaciones dominantes como el café y el banano, convirtiéndose en el sector productor más importante. Los datos más recientes indican que en el año 2017 Costa Rica recibió más de 2,96 millones de turistas, con un impacto económico cerca a los US\$ 3864 millones (Figura 1), generando el 35,4 \% de las divisas y el 6,7 \% del producto interno bruto (ICT, 2018a).

En 1996, el Instituto Costarricense de Turismo (ICT) diseñó e implementó el programa voluntario de Certificación para la Sostenibilidad Turística, conocido por las siglas "CST", para transicionar el concepto de sustentabilidad a algo real y práctico y así mantener la competitividad turística del país. Dicho programa, que ha sido descrito anteriormente (p. ej.: LePree, 2009; Vasconcelos-Vasquez et al., 2011; Tepelus \& Córdoba, 2005; Rivera, 2002; Vásquez \& Gutiérre, 2016) ha otorgado certificaciones para hoteles desde 1997, y hoy en día también certifica tour operadoras, empresas de alquiler de vehículos, y parques temáticos. El CST permite evaluar el desempeño de las empresas en cuatro ámbitos: entorno físico-biológico, planta de servicio (para establecimientos de hospedaje) o gestión del servicio (para agencias tour operadoras), cliente y entorno socioeconómico. Precisamente en este último ámbito es en que se enfoca este estudio, donde se evalúa la identificación e interacción de la empresa con las comunidades adyacentes, analizándose, por ejemplo, el grado en que las empresas turísticas responden al crecimiento y desarrollo de la localidad, mediante la generación de empleo o el logro de beneficios en pro de la colectividad. Esta certificación está reconocida internacionalmente por el Consejo Mundial de Turismo Sostenible (Global Sustainable Tourism Council, 2016), Rainforest Alliance y múltiples tour operadores emisores en Norteamérica y Europa (Spenceley y Bien, 2013; Valenzuela, Majano, Jäger y Kilian 2013).

\section{Turismo y su impacto local}

Localmente, el turismo puede contribuir al desarrollo comunitario, muy especialmente en áreas rurales (p. ej.: Fleischer \& Felsenstein, 2000). A través de sus múltiples modalidades (ecológico, rural, comunitario, de aventura), ha permitido inyectar y dinamizar el mercado, favoreciendo en gran parte a las zonas rurales donde se concentran muchas de las áreas protegidas (Mok 2005; Molina-Murillo, Hernández y Shreeve, 2016; White, 1993).

Aunque en este artículo usamos el término turismo sostenible para mantener concordancia con el nombre oficial del programa de certificación bajo análisis, este comparte los principios de turismo responsable discutidos por Idelhadj, Rivera \& Rodríguez (2012) quienes acercan la definición como aquella práctica integral que promueve aspectos como la reducción de la pobreza, el comercio justo, el trabajo decente, los salarios dignos, el respeto a la cultura e identidad de las comunidades locales, el enfoque de género, la participación y el desarrollo comunitario, la lucha contra problemas ambientales, el control y autogestión de los recursos naturales, así como la educación y sensibilización de los turistas hacia estos aspectos. Ellos mismos aclaran que el turismo responsable ha de ser necesariamente sostenible, pero va más allá, no como una categoría de turismo, sino como "un movimiento en favor de la sostenibilidad turística" (Cañada y Gascón, 2003: 98) que reconoce el papel central y el control que debe tener la población local sobre la actividad y su proceso de desarrollo, a partir de un señalamiento expreso de compromisos, sensibilidades y responsabilidades para los diferentes actores involucrados.

En comparación con el modelo turístico tradicional, el turismo responsable no solamente ayuda a conservar y proteger los atractivos y el patrimonio, sino que permite revalorizar las diversas manifestaciones culturales (Lane, 2011; Matarrita-Cascante, Brennan, \& Luloff, 2010), modelo que permite dar más control a la comunidad local sobre sus capitales (Bridger \& Luloff, 1999; De Rave, 2010, Molina y Clifton, 2014). En este sentido, se esperaría que desarrollar la actividad turística de una manera responsable permitiría promover la tenencia de la tierra de los pobladores locales, la utilización de prácticas productivas sostenibles (Vásquez \& Gutiérre, 2016), el fortalecimiento de la organización local (Briedenhann \& Wickens, 2004), y en última instancia, "la reducción de la pobreza en aquellas comunidades desfavorecidas" (Cordobés y Sanz, 2009). 
H1: Los hoteles certificados perciben un mayor impacto positivo sobre sus comunidades adyacentes que los hoteles no certificados

Según la teoría institucional, las organizaciones - para legitimarse - siguen una serie de reglas, creencias y prácticas prevalecientes en el sistema (DiMaggio \& Powell, 1983; Lowndes \& Roberts, 2013; Meyer \& Rowan, 1977); por tanto, si programas públicos voluntarios como el CST le permiten a los hoteles percibir una imagen positiva ante los diferentes actores con quien se relacionan para obtener dicha legitimidad, entonces, esta percepción sería clave para consolidar el éxito de dichos programas y la adhesión a sus principios inclusive en la eventual ausencia de ellos (Lyon \& Maxwell, 2007). Sin embargo, y a raíz del "efecto polizón" o "free rider problem" como se le conoce en inglés, algunas empresas se aprovecharon de la imagen natural que caracteriza a Costa Rica, defraudando a los visitantes con la promoción de productos turísticos "ecológicos" o "sustentables" sin que estos lo sean (Bien, 2002).

H2: Los hoteles buscan certificarse principalmente para alinearse a prácticas y normas prevalecientes en el sistema en busca de legitimización

\section{Metodología}

En esta investigación se utilizó un diseño transeccional (Hernández, Fernández y Baptista, 2010) para evaluar la percepción de hoteles costarricenses de su desempeño socioeconómico sobre su comunidad circundante. Primero se hizo una revisión de la literatura para establecer los principales indicadores socioambientales por evaluar, considerando los parámetros establecidos en evaluar la sostenibilidad turística como trabajadores, capacitación, interacción con la comunidad, beneficios económicos indirectos de la comunidad y desarrollo social y cultural (ICT, 2018b). Estos indicadores fueron discutidos en seis entrevistas a profundidad con dueños o administradores de hoteles certificados en las comunidades de Santa Elena y Monteverde alrededor del área protegida privada más visitada del país: la Reserva Bosque Nuboso Monteverde. Con la información obtenida se elaboró un cuestionario estructurado que luego se probó de manera piloto con encuestas presenciales en 35 hoteles en la zona turística de La Fortuna, alrededor del Parque Nacional Volcán Arenal. Después de unos pequeños ajustes para lograr mejor interpretación, se diseñó y aplicó en el año 2013 una encuesta por internet utilizando la plataforma SurveyMonkey.com. La muestra total se obtuvo a través de los hoteles afiliados en su momento a la Cámara Nacional de Turismo (356), y los 130 hoteles certificados CST del Instituto Costarricense de Turismo. Del total de 486 hoteles se recibieron 121 respuestas (24,9\% tasa de respuesta), para un error de muestreo del 7,73 a un $95 \%$ de confianza. La encuesta final se estructuró considerando datos generales del encuestado, datos generales del hotel, interacción del hotel con la comunidad y la influencia que tiene el hotel sobre la comunidad. En el caso de los hoteles con la certificación también se tomó el dato del número de estrellas y hojitas (análogo para el nivel de eco-certificación). La medición de estos elementos a través de estudios de percepción es valiosa ya que, como lo discuten Molina y Clifton (2014, p.84), individuos y comunidades actúan según su interpretación de la realidad, la cual está influencia tanto por elementos subjetivos como objetivos. El análisis de los datos se llevó acabo usando el programa estadístico JMP Pro10, utilizando análisis factorial y de medias no paramétricas debido al uso de escalas ordinales.

\section{Resultados}

Se obtuvo una muestra balanceada donde $51,2 \%$ de los hoteles poseen el certificado CST. El 64,6 \% de los hoteles son de capital nacional, el 23,9 \% extranjero y el restante de capital mixto. La media de operación fue de 59 años. Como se muestra en la Figura 2, los hoteles están distribuidos normalmente en cuanto al número de estrellas, y similarmente para aquellos certificados, en cuanto al número de hojitas galardonadas. 
Figura 2: Distribución de los hoteles muestreados según la calificación obtenida sobre la calidad en general y sostenibilidad turística

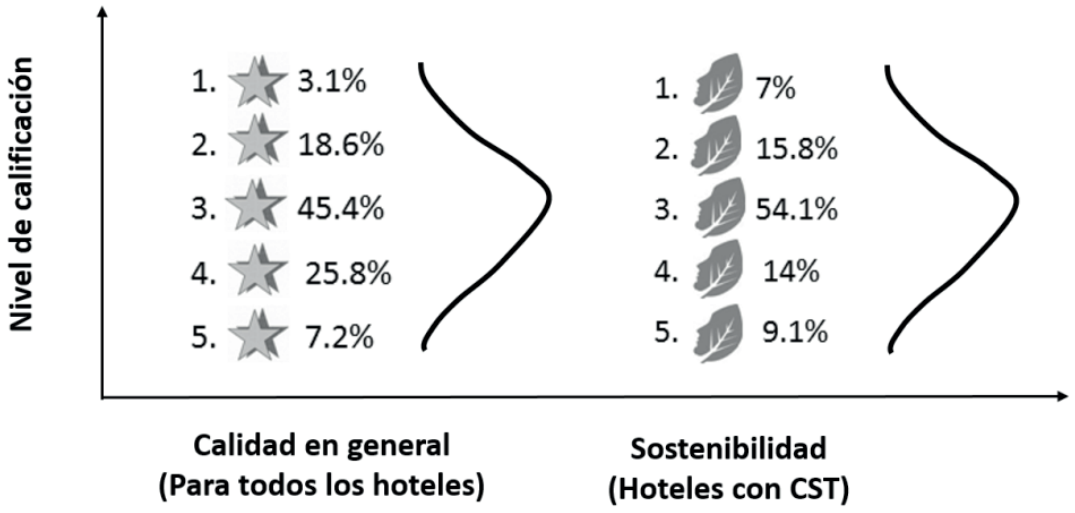

Fuente: Elaborado a partir de datos propios de la encuesta.

Antes de hacer la comparación sobre la percepción que los hoteles con y sin certificación CST tienen sobre los impactos socioeconómicos que ellos ejercen en sus comunidades circundantes, se procedió a realizar un análisis factorial confirmatorio para asegurar que efectivamente al analizar los impactos positivos y los negativos estaban siendo considerandos como dos dimensiones. La agrupación de los datos en ambos factores confirma dicha percepción (Cuadro 1); igualmente el indicador alfa de Cronbach mayor a 0,70 nos asegura la confiabilidad en dichos factores (Nunnally \& Berstein, 1994).

\section{Cuadro 1: Análisis factorial confirmatorio sobre componentes principales* de los impactos socioeconómicos que los hoteles tienen sobre sus comunidades circundantes}

\begin{tabular}{|l|c|c|c|}
\hline & Factor $\mathbf{1}$ & Factor 2 & a Cronbach $\boldsymbol{~}^{* *}$ \\
\hline Ayuda a mejorar la calidad de empleo e ingreso & 0,72 & $-0,04$ & 0,809 \\
\cline { 2 - 4 } Ha fortalecido la conciencia ambientalista & 0,67 & $-0,15$ & \\
Incentivan el aprendizaje del idioma inglés & 0,72 & 0,17 & \\
Incentiva participación de mujeres en puestos de liderazgo & 0,68 & $-0,02$ & \\
Ha promovido el desarrollo de infraestructura comunitaria & 0,71 & 0,16 & \\
Apoya en la capacitación y educación & 0,82 & 0,03 & \\
\hline Propicia mayor alcoholismo/drogadicción & $-0,11$ & 0,80 & 0,718 \\
Ha ocasionado aumento en la prostitución & $-0,17$ & 0,75 & \\
Ha causado un aumento de los precios en general & 0,13 & 0,69 & \\
Ha alterado la cultura tradicional & $-0,08$ & 0,56 & \\
Favorece el aumento de la población & 0,26 & 0,64 & \\
Ha favorecido la inseguridad ciudadana & 0,10 & 0,50 & \\
\hline Eingen values & 4,286 & 2,708 & \\
Porcentaje de la varianza explicada & 32,967 & 20,834 & \\
\hline
\end{tabular}

* Rotación varimax. ${ }^{* *}$ Confiabilidad del factor

Fuente: Elaborado a partir de datos propios de la encuesta. 
Examinar la percepción sobre los impactos socioeconómicos que los hoteles con o sin el Certificado para la Sostenibilidad Turística (CST) del Instituto Costarricense de Turismo pueden tener sobre sus comunidades es un elemento clave para entender el éxito de estos programas públicos voluntarios (Lyon \& Maxwell, 2007). En respuesta a la primera pregunta planteada de si perciben los hoteles certificados —en relación con los no certificados - una mayor generación de beneficios socioeconómicos a sus comunidades locales, se muestra que efectivamente tal diferencia de percepciones ocurre. Esto apoya nuestra primera hipótesis, aunque como se muestra en el Cuadro 2, los impactos socioeconómicos positivos fueron percibidos significativamente distintos, entre los hoteles certificados CST y aquellos que no lo están, donde la media de los primeros fue de 4,26 en una escala de 1 a 5.

La generación de capital económico como empleo, ingresos, infraestructura comunitaria, así como de capital social en la forma de aprendizaje de un segundo idioma, fortalecimiento de la conciencia ambiental y desarrollo de liderazgo en mujeres, son todos indicadores de que, al menos en la percepción de los hoteles certificados, el CST logra su objetivo de impulsar la sostenibilidad. Aunque no se cuenta con la información para asegurarlo, tal aseveración debe tomarse con cautela, ya que autores como Potoski \& Prakash (2013) han evidenciado que la participación en estos programas ocurre con frecuencia por empresas que ya de por sí eran superiores en estos parámetros de sustentabilidad.

En el caso de la percepción de los impactos socioeconómicos negativos sobre las comunidades, se observa que dicha diferencia no fue evidente estadísticamente, con ambos tipos de hoteles mostrando valores similares y menores a 2; acá podría estar ocurriendo un efecto halo, un sesgo cognitivo que desde la psicología interpreta que los hoteles podrían considerar que, por su razón de ser, en su mayoría generan solamente impactos positivos sobre sus comunidades.

\section{Cuadro 2: Diferencias en las medias entre hoteles con CST y hoteles no certificados en los impactos socioeconómicos positivos y negativos que ellos tienen sobre sus comunidades circundantes}

\begin{tabular}{|l|c|c|c|c|}
\hline & Media* & $\begin{array}{c}\text { Desviación } \\
\text { Estandar }\end{array}$ & $\begin{array}{c}\text { Valor } \\
\text { Z-Wilcoxon }\end{array}$ & Prob. \\
\hline Impactos socioeconómicos negativos & & & & \\
\hline Hotel certificado CST & 1,92 & 0,707 & $-0,690$ & 0,488 \\
\hline Hotel no certificado CST & 1,81 & 0,654 & & \\
\hline Impactos socioeconómicos positivos & & & & \\
\hline Hotel certificado CST & 4,25 & 0,532 & $-4,082$ & $<0,001$ \\
\hline Hotel no certificado CST & 3,66 & 0,846 & & \\
\hline
\end{tabular}

* Escala de 1 a 5, donde 1 es bajo impacto y 5 es alto impacto.

Fuente: Elaborado a partir de datos propios de la encuesta.

Cuando se consultó sobre como los hoteles interactúan más comúnmente con sus comunidades circundantes, se evidencia que todos los hoteles parecen percibir un alto nivel de interacción comunitaria. En general, la evaluación tiende a ser mejor para los hoteles certificados en comparación con aquellos no certificados (Cuadro 3). Estadísticamente este fue el caso para aquellos mecanismos de interacción más profundos como "participar activamente en eventos organizados por la comunidad", en la "compra de productos locales para uso en el hotel", o a través de "donaciones a grupos comunitarios" como equipamiento de cómputo o implementos deportivos. Si se considera que los dos mecanismos de interacción comunitaria más superficiales ("la generación de empleo" y "el impulso al desarrollo del turismo local") no ofrecen diferencias significativas, es difícil explicar que hoteles sin el CST estén interesados de adoptar dicha certificación cuando ya sienten que están apoyando su comunidad local. ¿Existen entonces otras razones por las cuales los hoteles estarían interesados en participar en dicho programa voluntario? 


\section{Cuadro 3: Comparación de los principales mecanismos de interacción de los hoteles sobre sus comunidades}

\begin{tabular}{|c|c|c|c|c|}
\hline \multirow{4}{*}{\begin{tabular}{|c} 
Mecanismo de interacción con la \\
comunidad
\end{tabular}} & \multicolumn{2}{|c|}{ CST } & \multirow{3}{*}{\multicolumn{2}{|c|}{$\begin{array}{c}\text { Valor } \\
\text { Z-Wilcoxon }\end{array}$}} \\
\hline & \multirow{2}{*}{$\begin{array}{c}\text { Sí } \\
\text { Med.* (D.E.) }\end{array}$} & \multirow{3}{*}{$\begin{array}{c}\text { No } \\
\text { Med. }(\text { D.E. })\end{array}$} & & \\
\hline & & & & \\
\hline & $4,16(0,88)$ & & $-2,4$ & $* *$ \\
\hline Ayuda a impulsar el turismo local & $4,26(0,81)$ & $3,96(1,08)$ & $-1,03$ & \\
\hline Favorece la compra de productos locales & $4,66(0,61)$ & $4,17(1,01)$ & $-2,37$ & $* *$ \\
\hline Genera empleo en la comunidad & $4,79(0,49)$ & $4,54(0,78)$ & $-1,58$ & \\
\hline Hace donaciones a grupos comunitarios & $4,42(0,78)$ & $3,88(1,19)$ & $-1,99$ & $* *$ \\
\hline
\end{tabular}

* Escala de 1 a 5 , donde 1 es bajo impacto y 5 es alto impacto. ** (Prob. $\leq 0.05)$

Fuente: Elaborado a partir de datos propios de la encuesta.

$\mathrm{Al}$ analizar sobre las razones principales por las cuales los hoteles han adoptado o adoptarían participar en el programa Certificado para la Sostenibilidad Turística (CST) no evidencia apoyo a la segunda hipótesis. Los resultados indican que la principal razón es ser más ecoeficiente, seleccionado por el $90 \%$ de los hoteles ya sea como primera, segunda o tercera opción (Cuadro 4), aspecto alineado con la adopción por parte de los hoteles de una estrategia de responsabilidad social empresarial (Vásquez, Balbastre-Benavent, \& Redondo-Cano, 2011; Vásquez \& Gutiérre, 2016). Capturar clientes con conciencia ambiental es la segunda razón de importancia, considerada como la principal segunda opción, con un $44 \%$ de los hoteles, siendo esta una razón estratégica sobre el negocio y no seriamente integrada a una estrategia de educación ambiental por parte de los hoteles (Stem, Lassoie, Lee, \& Deshler, 2003). Mejorar la relación con el Gobierno o con la comunidad parece un beneficio tangencial, y aunque cerca de un tercio de los hoteles lo considera como una tercera opción, solamente para un $2 \%$ de ellos lo señala como una opción prioritaria. Esto supera la propuesta de la teoría institucional sobre participar en el programa simplemente para obtener una legitimación (DiMaggio \& Powell, 1983; Lowndes \& Roberts, 2013) y evidencia que efectivamente el programa CST ofrece a los hoteles suficientes incentivos excluibles, aspecto que Potoski \& Prakash (2013) señalan como clave para su éxito.

\section{Cuadro 4: Razones por las cuales el hotel ha adoptado o adoptaría el CST}

\begin{tabular}{|l|c|c|c|}
\hline \multicolumn{1}{|c|}{ Razones } & \multicolumn{3}{c|}{ Nivel de prioridad } \\
\hline Ser más ecoeficiente & $\mathbf{1}^{\text {era }}$ opción & $\mathbf{2}^{\text {da }}$ opción & $\mathbf{3}^{\text {ra }}$ opción \\
\hline Capturar clientes con conciencia ambiental & $56 \%$ & $25 \%$ & $9 \%$ \\
\hline Porque es lo correcto/ filosofía empresarial & $17 \%$ & $44 \%$ & $22 \%$ \\
\hline Porque otros hoteles tienen la certificación & $11 \%$ & $2 \%$ & $0 \%$ \\
\hline Reducir los costos de operación & $7 \%$ & $4 \%$ & $7 \%$ \\
\hline Mejorar relaciones con el Gobierno y comunidad & $2 \%$ & $19 \%$ & $33 \%$ \\
\hline Otro & $2 \%$ & $6 \%$ & $27 \%$ \\
\hline Total & $6 \%$ & $0 \%$ & $2 \%$ \\
\hline
\end{tabular}

Fuente: Elaborado a partir de datos propios de la encuesta.

Finalmente, se aclara que también se evaluaron los impactos socioeconómicos positivos y negativos tanto para los hoteles por nivel de desempeño general (estrellas) como por el desempeño en sostenibilidad (hojitas) pero no se encontraron diferencias significativas. Adicionalmente, se hizo un análisis de contingencia para determinar la presencia de alguna relación entre el número de estrellas y si el hotel está o no certificado. 
Aunque se muestra una pequeña tendencia de hoteles certificados CST con más 4 y 5 estrellas, o de hoteles no certificados CST con más 1 y 2 estrellas, en realidad la mayoría de datos se concentran sobre la media (3 estrellas) y provoca que no haya diferencias significativas. Estos resultados indican que existe un interés por obtener la certificación de sostenibilidad independientemente del nivel de la calidad de los servicios e instalaciones del hotel, aunque dicha calidad con frecuencia está limitada por un factor presupuestario.

\section{Conclusiones}

Costa Rica a través del Certificado para la Sostenibilidad Turística (CST) ha desarrollado un programa con alto potencial para incentivar a que hoteles y otras opciones de hospedaje diseñen y ejecuten sus estrategias de negocio de manera responsable. A pesar de las posibles deficiencias que el programa pueda tener, en este estudio se demuestra que el CST influye de manera favorable en la percepción de los impactos socioeconómicos positivos que los hoteles tienen sobre la comunidad donde operan. Hoteles certificados tienden a interrelacionarse con sus comunidades circundantes de una manera que va más allá de su razón de ser - la de hacer dinero- a participar en eventos comunitarios, favorecer la compra de productos locales o inclusive a través de pura filantropía. No obstante, es evidente que para cualquier tipo de hotel — certificado o no- obtener dicho reconocimiento tiene un objetivo estratégico de negocio: ser más ecoeficiente y reducir costos, por tanto, los posibles impactos socioeconómicos sobre las comunidades locales donde operan parecieran ser efectos colaterales y no la razón principal para adoptar el CST. Se sugiere entonces, que el programa ponga más énfasis en facilitar el empoderamiento de las comunidades y su representación sociocultural desde la actividad turística (LePree, 2009).

Aunque el desempeño de un programa voluntario como este no debería medirse por el número de hoteles participantes, las barreras de entrada o los beneficios percibidos son fundamentales para adherir principalmente a aquellos actores que mayor necesidad de cambio requieren. Este estudio provee evidencia sobre esta mayor percepción de beneficios, un elemento central en la difusión de información y éxito de programas públicos voluntarios como este (Lyon \& Maxwell, 2007; Potoski \& Prakash, 2013). Actualmente el costo de verificación es gratuito; sin embargo, los costos de implementación para ejercer una actividad responsable de la manera como lo proponen Cañada y Gascón (2003) y Idelhadj, Rivera \& Rodríguez (2012) podrían ser significativos, empero, de mayor beneficio para la comunidad local y la sociedad en general.

Reconocemos que este estudio está limitado por un análisis comparativo en relación con la percepción que las comunidades puedan tener sobre los hoteles. Por tanto, una de las preguntas que se deriva de este análisis es: ¿perciben las comunidades de hoteles certificados, de manera agregada, mayores beneficios socioeconómicos? Aunque esta pregunta se ha explorado en otras latitudes (Andereck et al., 2005) o para un hotel en particular (Almeyda et al., 2010), para el programa CST la respuesta está aún por investigarse.

\section{Bibliografía}

Almeyda, A. M., Broadbent, E. N., Wyman, M. S., \& Durham, W. H.

2010. Ecotourism impacts in the Nicoya Peninsula, Costa Rica. International Journal of Tourism Research, 12(6), 803-819. https://doi.org/10.1002/jtr.797

Andereck, K. L., Valentine, K. M., Knopf, R. C., \& Vogt, C. A.

2005. Residents' perceptions of community tourism impacts. Annals of Tourism Research, 32(4), 1056 -1076. https://doi.org/10.1016/j.annals.2005.03.001

Bien, A.

2002. Environmental Certification for Tourism in Central America: CST and Other Programs. In Honey, M.: Ecotourism and Certification: Setting Standards in Practice. Washington: Island Press.

Bridger, J. C., \& Luloff, A. E.

1999. Toward an interactional approach to sustainable community development. Journal of Rural Studies, 15(4), 377-387. https://doi.org/10.1016/S0743-0167(98)00076-X

Briedenhann, J., \& Wickens, E.

2004. Tourism routes as a tool for the economic development of rural areas-vibrant hope or impossible dream? Tourism Management, 25(1), 71-79. https://doi.org/10.1016/S0261-5177(03)00063-3

Buckley, R.

2012. Sustainable tourism: Research and reality. Annals of Tourism Research, 39, 528-546. https://doi. org/10.1016/j.annals.2012.02.003

Cañada, E. y Gascón, J.

2007. Turismo y Desarrollo: Herramientas para una mirada crítica. Managua: Enlace. Disponible en http://www.albasud.org/downloads/142.pdf 
Cordobés, M. y Sanz, B. (Coord.)

2009. Turismo para el Desarrollo. Barcelona: Obra Social. Fundación "la Caixa". Disponible en https:// multimedia.caixabank.es/lacaixa/ondemand/obrasocial/agendaelectronica/cooperacioninternacional/ turismo/pdf/turismo_para_el_desarrollo.pdf

De Rave, K.

2010. Estudio de caso sobre los requisitos del programa Certificado de Sostenibilidad Turística (CST) del Instituto Costarricense de Turismo (ICT) para posadas de turismo rural. Tesis como requisito para optar por el título de máster en gestión de Turismo Sostenible. UCI. San José, CR. 106p.

DiMaggio, P. J., \& Powell, W. W.

1983. The Iron Cage Revisited: Institutional Isomorphism and Collective Rationality in Organizational Fields. American Sociological Review, 48(2), 147-160. https://doi.org/10.2307/2095101

Fleischer, A., \& Felsenstein, D.

2000. Support for rural tourism: Does it make a difference? Annals of Tourism Research, 27(4), 1007 -1024. https://doi.org/10.1016/S0160-7383(99)00126-7

Flores, A.

2014. Compendio de los logros más importantes del Ministro de Turismo en el periodo 2011-2014 (Informe final de gestión). Instituto Costarricense de Turismo. Disponible en http://www.ict.go.cr/es/documentos-institucionales/departamento-de-recursos-humanos/informes-finales-de-gesti\%C3\%B3n/455-informe-final-de-labores-lic-allan-flores-moya,-ex-ministro-de-turismo/file.html

Global Sustainable Tourism Council

2016. List of Recognized Standards for Hotels \& Tour Operators. Disponible en https://www.gstcouncil. org/en/gstc-criteria-hotels-tour-operators-destinations/gstc-recognized-standards/gstc-recognized-standards-for-hotels.html

Hernández, R.; Fernández, C., \& Baptista, P.

2010. Metodología de la Investigación. 5ta Ed. México: McGraw-Hill

Honey, M.

2003. Giving a Grade to Costa Rica's Green Tourism. NACLA Report on the Americas, 36(6), 39-47.

Horton, L. R.

2009. Buying Up Nature: Economic and Social Impacts of Costa Rica's Ecotourism Boom. Latin American Perspectives, 36(3), 93-107.

Hunt, C. A., Durham, W. H., Driscoll, L., \& Honey, M.

2015. Can ecotourism deliver real economic, social, and environmental benefits? A study of the Osa Peninsula, Costa Rica. Journal of Sustainable Tourism, 23(3), 339-357. https://doi.org/10.1080/096 69582.2014.965176

Idelhadj, A., Rivera Mateos, M., \& Rodríguez García, L.

2012. Turismo responsable, espacios rurales y naturales y cooperación para el desarrollo: a propósito de la "Declaración de Tetuán" (Marruecos). PASOS Revista de turismo y patrimonio cultural, 10(5), 651-664. https://doi.org/10.25145/j.pasos.2012.10.079

Instituto Costarricense de Turismo (ICT)

2018a. Anuario Estadístico de Turismo 2018: Divisas por concepto de turismo. Disponible en http:// www.ict.go.cr/es/estadisticas.html

Instituto Costarricense de Turismo (ICT)

2018b. Certificación para la Sostenibilidad Turística. Disponible en http://www.turismo-sostenible.co.cr/ Instituto Nacional de Biodiversidad (INBio).

2016. Biodiversidad en Costa Rica. (En línea). Disponible en: http://www.inbio.ac.cr/conservacion.html Lane, B.

2011. Sustainable rural tourism strategies: A tool for development and conservation. Revista Interamericana de Ambiente Y Turismo - RIAT, 1(1), 12-18. https://doi.org/10.4067/riatvol1iss1pp12-18\%0718-235X LePree, J.G.

2009. Certifying Sustainability: The Efficacy of Costa Rica's Certification for sustainable tourism. Florida Atlantic Comparative Studies Journal 11: 57-78.

Lindberg, K., \& Aylward, B.

1999. Price Responsiveness in the Developing Country Nature Tourism Context: Review and Costa Rican Case Study. Journal of Leisure Research, 31(3), 281-299.

Lowndes, V. \& Roberts, M.

2013. Why institutions matter. Hampshire: Palgrave Macmillan.

Lyon, T. P., \& Maxwell, J. W.

2007. Environmental Public Voluntary Programs Reconsidered. Policy Studies Journal, 35(4), 723-750. https://doi.org/10.1111/j.1541-0072.2007.00245.x

Matarrita-Cascante, D., Brennan, M. A., \& Luloff, A. E.

2010. Community agency and sustainable tourism development: the case of La Fortuna, Costa Rica. Journal of Sustainable Tourism, 18(6), 735-756. https://doi.org/10.1080/09669581003653526 
Meyer, J. W., \& Rowan, B.

1977. Institutionalized Organizations: Formal Structure as Myth and Ceremony. American Journal of Sociology, 83(2), 340-363. https://doi.org/10.1086/226550

Mok, C.S.

2005. Turismo y ambiente: un potencial para el desarrollo económico para Costa Rica. Reflexiones 84 (2): 25-37.

Molina, S. A., \& Clifton, J.

2014. Perceptions of institutions and development in rural Costa Rica. The Journal of Rural and Community Development, 9(4): 80-101. http://journals.brandonu.ca/jrcd/article/view/898

Molina-Murillo, S., Hernández, M., \& Shreeve, K.

2016. Understanding the link between benefits from protected areas and its relationship with surrounding communities: An exploration in Costa Rica. Parks Journal, 22(1):77-86. http://dx.doi.org/10.2305/ IUCN.CH.2016.PARKS-22-1SAM-M.en

Murray, G.

2007. Constructing Paradise: The Impacts of Big Tourism in the Mexican Coastal Zone. Coastal Management, 35(2-3): 339-355. http://doi.org/10.1080/08920750601169600

Nunnally, J. C., \& Berstein, I. R.

1994. Psychometric theory (3rd Edition). New York: McGraw-Hill. 752p.

Potoski, M., \& Prakash, A.

2013. Green Clubs: Collective Action and Voluntary Environmental Programs. Annual Review of Political Science, 16(1), 399-419. https://doi.org/10.1146/annurev-polisci-032211-211224

Rivera, J.

2002. Assessing a voluntary environmental initiative in the developing world: The Costa Rican Certification for Sustainable Tourism. Policy Sciences 35, 333-360.

Smith, M.

2015. Tourism and Cultural Change. En The Routledge Handbook of Tourism and Sustainability, Hall, M., Gossling, S., Scott, D. (Eds.) p. 175-184. New York: Routledge.

Spenceley, A. \& Bien, A.

2013. Ecotourism Standards: International Accreditation and Local Certification and Indicators. In: R. Ballantyne and J. Packer (ed.) International Handbook on Ecotourism, p. 404. Cheltenham, UK: Edward Elgar Publishing.

Stem, C. J., Lassoie, J. P., Lee, D. R., \& Deshler, D. J.

2003. How "Eco» is Ecotourism? A Comparative Case Study of Ecotourism in Costa Rica. Journal of Sustainable Tourism, 11(4), 322-347. https://doi.org/10.1080/09669580308667210

Tepelus, C. M., \& Córdoba, R. C.

2005. Recognition schemes in tourism-from 'eco' to 'sustainability'? Journal of Cleaner Production, 13(2), 135-140. https://doi.org/10.1016/j.jclepro.2003.12.015

Valenzuela, A.; Majano, A.; Jäger, U. \& Kilian, B.

2013. La política de turismo sostenible en Costa Rica como fuente de innovación para servicios de hospitalidad: Innovación y Productividad en el Sector Servicios. INCAE Business School. Alajuela, CR. $53 \mathrm{p}$.

Vasconcelos-Vasquez, K., Balbastre-Benavent, F., \& Redondo-Cano, A.

2011. Is certification for sustainable tourism complementary to ISO 9000 certification? The case of the Parque del Lago Hotel in Costa Rica. PASOS: Revista de Turismo y Patrimonio Cultural, 9(4), 543-557. http://www.pasosonline.org/Publicados/9411/PS0411_05.pdf

Vásquez, K. L. V., Balbastre-Benavent, F., \& Redondo-Cano, A. M.

2011. La certificación de sostenibilidad turística (CST): facilita la certificación ISO 9000: el caso del HPL. Revista de Ciencias Económicas, 29(1). Recuperado a partir de http://revistas.ucr.ac.cr/index. $\mathrm{php} / \mathrm{economicas} /$ article/view/7060

Vásquez, K. L. V., \& Gutiérre, C. M.

2016. Hotel Finca Campestre Rosa Blanca: Ejemplo de responsabilidad social empresarial en Costa Rica. Relaciones Internacionales, 89(1), 95-121. https://doi.org/10.15359/ri.89-1.5

White, D.

1993. Tourism as economic development for native people living in the shadow of a protected area: A North American case study. Society \& Natural Resources, 6(4), 339-345. https://doi. org/10.1080/08941929309380832

Recibido:

$26 / 12 / 2016$

Reenviado:

$27 / 06 / 2018$

Aceptado:

$21 / 12 / 2018$

Sometido a evaluación por pares anónimos 